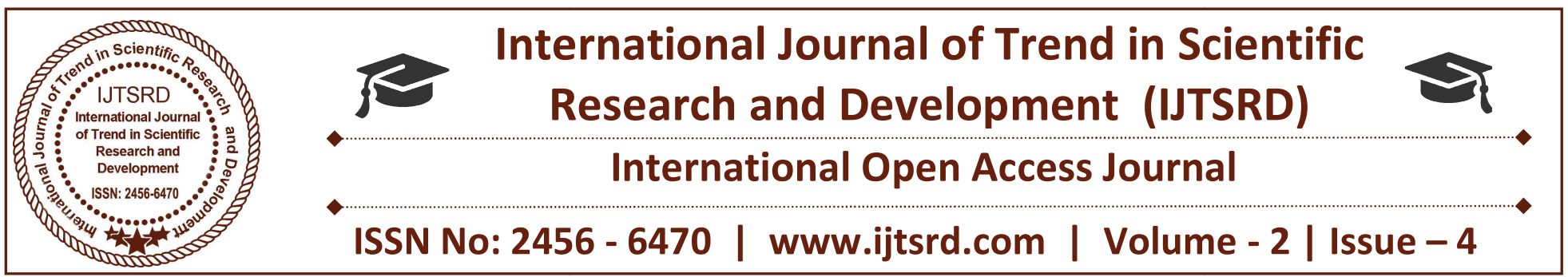

\title{
Analysis of Windshield Repair Technology
}

\author{
Abonyi Sylvester Emeka \\ Nnamdi Azikiwe University, Awka, Anambra State
}

\begin{abstract}
This paper presents analysis of different types of breaks in windshield and the repair process. Almost all windscreen damage can be attributed to stones or pebbles dislodged from tyres of vehicles in front, or simply drop from trucks carrying gravel. This object hit windscreen surface at great speed and the impact breaks the glass surface at the point of impact. Unfortunately, it may cause internal fracture of the glass, in which case a break occurs. The most common break one will see in windshield include: star shape, bullseye, round shape, cracks and combination breaks. These breaks cause blurred vision to drivers and can result in an accident. Replacing windshield is not cost effective and causes water linkages with time; hence repairing the crack becomes very necessary. To repair crack, resin with the same refractive index with the windshield is used. A follow-up paper will discussed the analysis of resins used in windshield repair.
\end{abstract}

Keywords: windshield, cracks, resin

\subsection{INTRODUCTION}

The first automobiles utilized plain sheet glass in a metal frame. It was learned very soon that some types of safety glass construction was necessary for passenger safety. In this process, the glass was formed and cooled very rapidly, setting up high compression forces in the outer layer of the glass. When tempered glass is hit by a projectile, it immediately shatters into very small granular pieces. This resulted in injuries to the occupants of the vehicle. Tempered glass is used today for side and rear windows. In the early time vehicle used tempered glass as Windshields, but now dual plate laminated safety glass is used as the front windshield. Auto glass (windshield) repair began officially in 1972 with the production of the first equipment and chemicals that were specifically designed to repair impact damages on the laminated windshield.

\subsection{Problems Associated with Replacement of Windscreen}

Original factory fitted windscreens require a minimum force of 1500 pounds per sq inch (psi) to detach it from the car body. Once the original seal is destroyed and a replacement windscreen installed, that strength is no longer guaranteed. Improper installation can be a result of a dozen or more causes ranging from improper handling, contamination of surfaces, wrong adhesives, inadequate drying or setting, etc. The result could lead to water leakage, air entry and worse, rust and corrosion which you don't get to see until it is too late.

Also it supports the structural integrity of the car especially in the area of roof support. That level of integrity is maintained with the original factory fitted windscreen. Removing the original and installing a new one opens up a multitude of potential problems. In other words, many things can go wrong, giving rise to a suspect installation.

\subsection{Analysis of Auto Glass Break}

There are two basic causes of broken windshield:

i. Impact: This is the most common break. It occurs when an object hit the windshield.

ii. Twist or Stress: This crack occurs when the windshield is twisted, either by flexing in the vehicle frame or because of improper mounting. It can be helped along by a nick in 
the edge of the windshield. When there is a break in laminated windshield, an air gap appears in the glass, resulting in surface inside the air gap, on either side. Also, there may be crushed glass inside the break, depending on the kind of break. For example the impact crack is much likely to have some crushed glasses inside the break.

When the outer layer of the glass is broken, the air space created in the crack causes random refraction of light. This air space appears as dark spots and may be distracting the driver. The objective of the process is to completely fill the air space with resin down to the plastic interlayer thus completely filling the voids and the cracks. Since the resin is carefully matched with to the optical properties of the glass, the break appears nearly invisible. The resin upon curing bonds the entire glass together resulting in a structurally sound area that is resistance to further cracking.

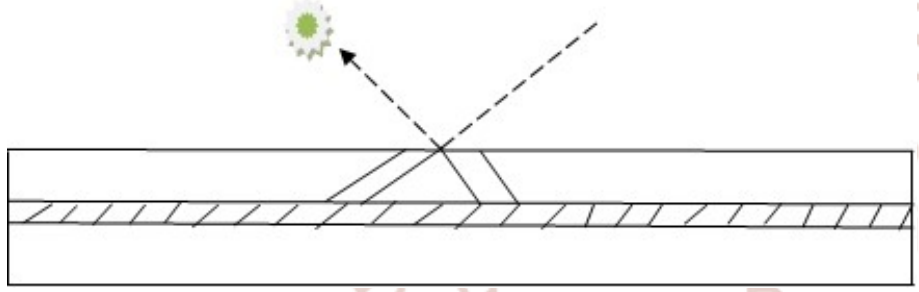

Fig. 1... windshields must have PVB thickness of not less than 0.030 inch. For all other uses, the PVB thickness can be 0.015 inch. Most times 0.030 inch laminated glass is referred to as "plate" and 0.015 inch laminated glass is referred to as "sheet." It is the PVB that qualifies a glass as safety glass. It enables the windshield to cushion the impact and help avoid serious injury. That is the PVB holds the broken glass and form a barrier to prevent the occupants from being thrown through the windshield. If the two layers of the glass break, the glass shards adhere to the PVB rather than showering the occupants with potentially life threatening pieces of jagged glass

Laminated glass does an equally good job of keeping objects thrown at the vehicle's windshield or kicked up from the road from penetrating the vehicle's passenger compartment. When an object cause cracks or various types of break on the outer lite of the windshield or other laminated glass part - that windshield/part becomes a potential candidate for repair.

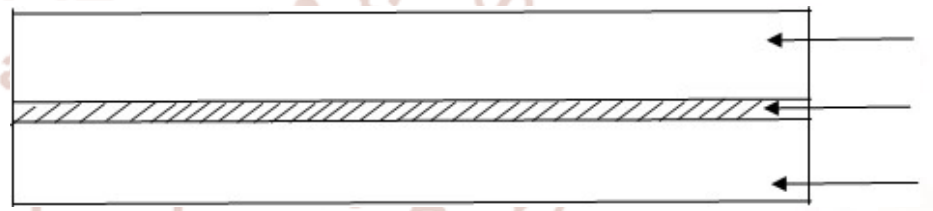

Fig. 1.2 Diagram of a laminated glass

\subsection{Types of Auto Glass and Component}

There are two main types of automotive safety glass in use today. They include;

(a) Laminated glass

(b) Tempered glass

Although both of the glass types are considered safety glass they each have different job to do. Laminated glass helps to keep the occupants inside the vehicle safe in case of collision. Laminated glass is used primarily for windshields, but can be used in other auto glass part.

Laminated glass consists of two sheets of float glass with a sheet of plastic (ply vinyl butyral) sandwiched as shown in Fig. 1.2. According to federal standard, 
International Journal of Trend in Scientific Research and Development (IJTSRD) ISSN: 2456-6470

\subsection{Break identification}

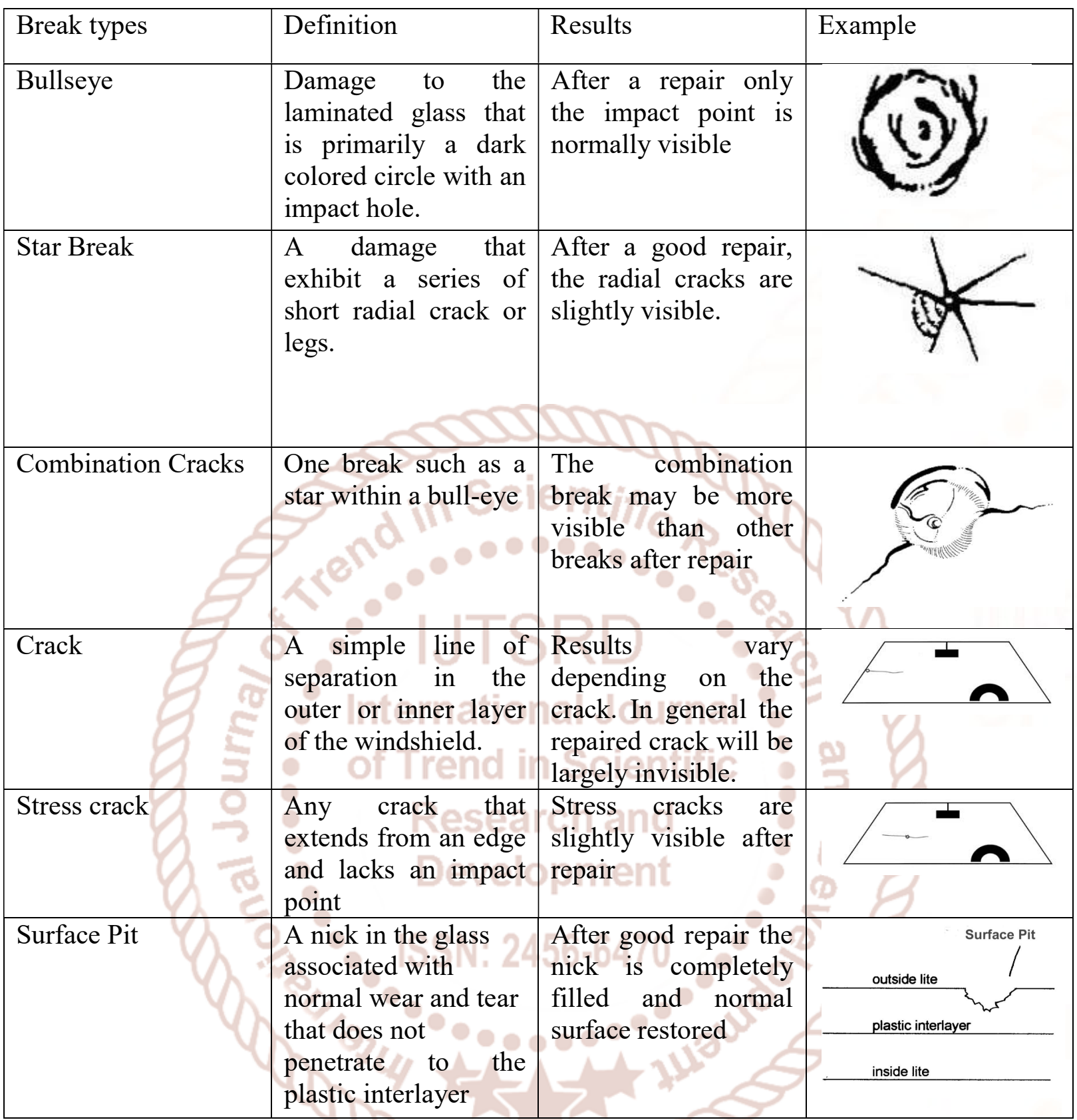

Table.1 Identification of Breaks in windshield

\subsection{Damage Types and Repairable Dimensions}

There are different types of crack one will encounter in repairing windshield. These may include,

i. Short crack - A crack on the windshield of 6 inches $(25.24 \mathrm{~cm})$ in length or less

ii. Long crack - A crack on the windshield of more than 6 inches in length

iii. Horizontal crack - the most common crack

iv. Vertical crack - the tightest crack

v. L-Shape Crack - the second most common crack vi. Edge Crack - Any crack on the windshield that extend to the edge

vii. Floating Crack - Any crack on the windshield that does not extend to the edge

viii. Stress Crack - Any crack that extends from the edge without impact point.

ix. Star break - Diameter of the break not to exceed 3 inches $(75 \mathrm{~mm})$

x. Bullseye - the most difficult to repair, With a diameter no larger than one inch (25 mm)

xi. Surface Pit: Damage with a diameter of not less than $1 / 8$ inch $(3 \mathrm{~mm})$ 
International Journal of Trend in Scientific Research and Development (IJTSRD) ISSN: 2456-6470

xii. Combination break - Diameter of body (excluding legs) not to exceed 2 inches (50 $\mathrm{mm})$

\subsection{Repair Limitations}

Both the location and the condition of the damage are important considerations in the decision to repair. Replacement is recommended under any of the following circumstances, i.e. do not repair:

i. damage that penetrates both the inside and outside layer of a laminated glass

ii. damage with three or more long cracks emanating from a single impact point

iii. damage on the inside lite (layer) of laminated glass

iv. damage contaminated with visible impurities that cannot be removed through cleaning.

v. damage or discoloration to the plastic interlayer

vi. damage in an area of the windshield where value-added features may be

vii. negatively affected by the damage and/or the repair process

viii. damage with a pit size greater than $3 / 8$ inch $(9 \mathrm{~mm})$

ix. edge $\operatorname{crack}(\mathrm{s})$ that intersect more than one edge

x. $\quad$ stress cracks in the Driver's Primary Viewing Area (DPVA), if diameter of damage is larger than one inch $(25 \mathrm{~mm})$.

\subsection{Repair Setting Tools}

Windscreen repair kits are used for repairing these breaks in our windshield. The repair kits started with the flagship Maxim Repair System to new Spectrum windscreen repair kit both using PRISM technology.Windshield Setting Tools are designed to promote safety for the technician and safe installations for consumers. The repair tools includes;

i. Power mounting bracket or PVB (power Vac)

ii. Power Vac injection body

iii. Power VacPistion

iv. Power Vac injection base seal

v. Power Vac injection pressure seal

vi. Piston control screw

vii. Ultra violet sun shade or U-V sun shade

viii. U-V curing lamp

ix. Crack exopander $\mathrm{x}$. Dremel for drilling

xi. Drill bits collect

xii. Polish wheel collect

xiii. Carbide pick

xiv. Injector cleaner

XV. Advance spot heater

xvi. Magnified visor (lens)

xvii. Blades

xviii. Curing films

xix. Curing strips

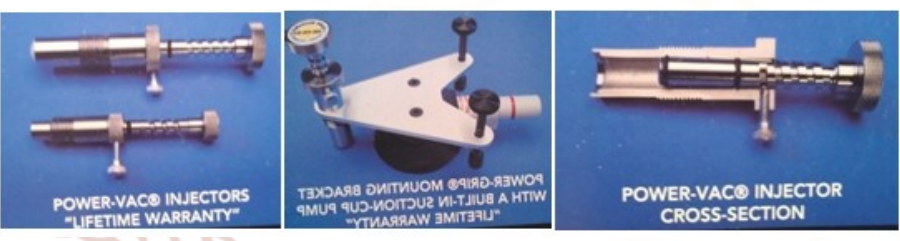

Fig 3 (Courtesy of glass mechanix USA)

\subsection{Repair Process}

A windscreen crack tends to run with the slightest of pressure or temperature change and usually originates from a break at the windscreen edge. Breaks can come in more complicated forms, taking shape in a multitude of designs. They may require extreme effort in repairing and hence take a longer time to do. Due to the complexity of the damage, such breaks are generally termed Complex breaks.

A web like break that can cover half your windscreen, this break is typically caused by a rounded surface striking the glass surface with impact. It may be caused by a child's head, a golf ball, a baseball bat or a crash helmet. Repair is not recommended in such cases, due to the extent of the damage. They represent less than $1 \%$ of the typical damage we see on windscreens.

Windscreen chips are the most common form of damage one can find on a windscreen. A chip results when a piece of glass is removed upon impact with a stone or pebble, with no internal fracture or break in the glass. Only surface repair is required in such a case. If left unrepaired, such chips will cause wiper blade damage

For best result, repair should be done soon after the damage occur. The windshield should be shaded from direct sunlight until the entire repair is complete. Windshield breaks can be classified into two major groups, namely: 


\section{Chips, Bull's Eye and Star Break}

Step 1

For chips, bull's eye and star cracks, remove loose glass fragments by scraping over with the razor blade. Affix the applicator base to the windshield. Make sure the center ring is centered above the break andsuction cups are firmly attached to the windshield. Do not allow the suction cups to cover any cracks extending from the central point.

Step 2

Screw the resin chamber into the center ring. If necessary re-maneuver the device so that the rubber mouth will be directly on top of the point of impact. Check the alignment from inside the car.

Screw down the resin chamber (turning clockwise) so the rubber mouth is flat against the windshield, in complete but gentle contact with the glass. Excessive pressure will alter the original curve on the glass, affecting the flow of the repair resin and effectiveness of the repair.

Step 3

Squeeze 3-6, drops of repair resin (depending on the extent of the damage) into the resin chamber mounted on the windshield. If the resin leak out of the bottom of the resin chamber, gently turn the resin chamber anti-clockwise just enough to prevent the leakage.

Step 4

Screw the pressure driver (clockwise) into the resin chamber to drive the resin into the break. Tighten until the pressure driver is almost screwed all way in. check from inside car to make the rubber has opened wide. Allow the repair rein time to be absorbed into the crack (2-3 minutes). If the resin has not reached all the areas of break, apply heat from inside the windshield with hot air gun. Caution should be applied when using the hot air gun as excessive heat can cause damage. Slightly retighten the pressure driver (about 1/4 turn) to push the resin further into the break.

Step 5

Unscrew and remove the pressure driver from the resin chamber to release any air trapped in the chamber. Re-insert the pressure driver and screw down again to displace any air remaining in the break. To determine if the repair resin has been absorbed into the break, look at the glass from an angle of about $45^{\circ}$. Make sure no air bubbles remain in the break. In the case of Chips or Bull's eye, examine the glass from directly behind the damaged area. If there are any damage still visible, repeat steps 4 and 5 until satisfactory result is achieved. Make sure there enough resin remaining in the resin chamber; if necessary add more repair resin.

Step 6

After the scripts have been removed, turn the resin chamber and pressure driver together half a turn to the left (counter clockwise). Remove the entire apparatus by lifting up on small tab of the suction cups. Gently wipe away the excess repair resin from the applicator so it does not drip down the windshield. Add a drop of the repair resin onto areas where damage is still visible and cover with curing strips for the finishing touches. When applying the curing strips to the repair, no pressure is necessary, gently place the strips on the glass.

\subsection{Conclusion}

The Windshield repair Technology's goal is to provide the best crack repairs possible. It was believed that the secret to a quality long crack repair was in the resin and filling process. The development of more sophisticated equipment and superior resins has enabled the repair to be economically, environmentally and physically sound process which, when properly performed. This has resulted in:

I. Restore strength of the windshield

II. Prevent the original damage from spreading further

III. Retain the original seal and save the windshield

IV. Improve the optical clarity in the damaged area and dramatically improve the cosmetic appearance of the glass by 70 to $95 \%$.

V. Create a smooth surface to prevent interference with the windshield wipers.

The subsequent paper will address the analysis of the resins that are used for the windshield repair. The resin is the chemical substance that bonds the broken glass. 
International Journal of Trend in Scientific Research and Development (IJTSRD) ISSN: 2456-6470

\section{References}

1) Instruction manual, Glass mechanix

2) Windshield Repair Manual For Super Cinch www.GlassProSystems.Com

3) American Windshield Repair Systems. Website: www.rockchipkits.com

4) www.rainx.com
5) www.atuoglasssystem.com

6) www.wurthusa.com

7) Blue star Windshield Repair Systems Instruction manual www.bluestarproducts.com

8) www.glassmechanix@glassmechanix.com

9) Www.aegistools.com

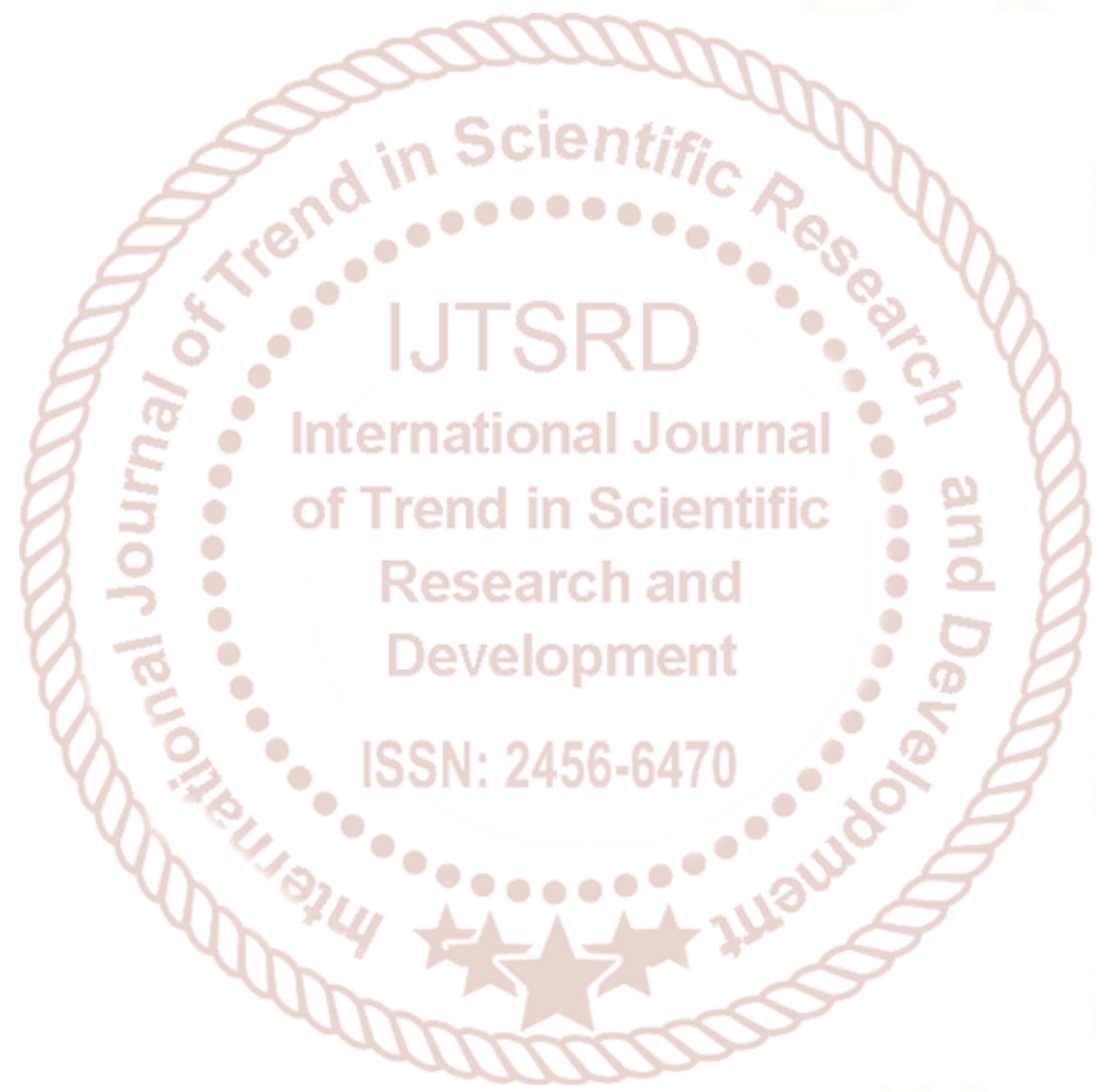

